Check for updates

Cite this: RSC Adv., 2017, 7, 47758

Received 27th June 2017

Accepted 26th September 2017

DOI: $10.1039 / \mathrm{c} 7 \mathrm{ra0} 07110 \mathrm{~h}$

rsc.li/rsc-advances

\section{Licorice extract attenuates brain aging of D- galactose induced rats through inhibition of oxidative stress and attenuation of neuronal apoptosis $\uparrow$}

\author{
Yu-Zhi Zhou, (D) *a Fan-Fan Zhao, ${ }^{\text {ab }}$ Li Gao, ${ }^{a}$ Guan-Hua Du, ${ }^{\text {ac }}$ Xiang Zhang ${ }^{\text {ad }}$ \\ and Xue-Mei Qin*a
}

Licorice, an edible and officinal plant, has attracted considerable attention for its wide range of pharmacological activities. The present study focused on the protective effect of licorice on aging rats and on exploring its potential mechanisms. Aging in rats was induced by $D$-gal $\left(300 \mathrm{mg} \mathrm{kg}^{-1}\right)$ for 5 weeks continuously with intraperitoneal injection, while control rats received physiological saline, and other groups received different concentrations of licorice extract (1,5 and $10 \mathrm{~g}$ of herb per $\mathrm{kg}$ ) by oral gavage after being $\mathrm{D}$-gal induced. Their spatial and learning memory abilities were evaluated by the Morris water maze test and an object-place recognition test. Some related biochemical indices were also determined by assay kits. Neuronal cell injury in the hippocampal region was observed by hematoxylin and eosin (HE) staining. The expression levels of Bax, Bcl-2, caspase-3 and Cyt- $C$ in the hippocampus were detected by western blot. The results showed that licorice extract could significantly ameliorate the learning and memory abilities impaired by D-gal. The licorice extract significantly increased the SOD, GSH-Px and CAT activity and synchronously decreased the content of MDA in the hippocampus. It also ameliorated the dysfunction of the cholinergic system induced by $\mathrm{D}$-gal. Furthermore, the licorice extract also protected the hippocampal neurons against damage and could significantly reduce the expression of the $\mathrm{Bax} / \mathrm{Bcl}-2$ protein ratio, caspase- 3 and $\mathrm{Cyt}-\mathrm{C}$ protein in the hippocampus. These findings indicate that the licorice extract effectively attenuated cognitive damage, improved oxidative stress and apoptosis in aging rats induced by $\mathrm{D}$-gal, and played a significant role in antioxidation and anti-neuronal apoptosis.

\section{Introduction}

Licorice, an edible and officinal plant with a pleasing flavor, is the root or stem of Glycyrrhiza glabra (G. glabra). Because of its sweet favor, licorice is widely used in beer, beverages, candy, ice cream, baked cookies, soy protein imitation meat products, soy sauce and other foods, and it is also used in tobacco and cosmetics. ${ }^{\mathbf{1}, 2}$ As a traditional Chinese medicine, licorice exhibits various pharmacological effects and biological functions.

\footnotetext{
${ }^{a}$ Modern Research Center for Traditional Chinese Medicine, Shanxi University, No. 92 Wu Cheng Road, Taiyuan 030006, China.E-mail: zhouyuzhi@sxu.edu.cn; qinxm@ sxu.edu.cn; Fax: +86-0351-7019178; +86-0351-7011202; Tel: +86-0351-7019178; +86-0351-7011202

${ }^{b}$ College of Chemistry and Chemical Engineering, Shanxi University, Taiyuan 030006, China

'Institute of Materia Medica, Chinese Academy of Medical Sciences, Peking Union Medical College, Beijing 100050, China

${ }^{d}$ Department of Chemistry, University of Louisville, Louisville, KY 40229, USA

$\dagger$ Electronic supplementary information (ESI) available. See DOI: 10.1039/c7ra07110h
}

Modern studies have indicated that licorice has excellent antioxidant activity. ${ }^{3-7}$ Studies have also demonstrated that licorice extract can markedly increase the activities of liver superoxide dismutase (SOD), catalase (CAT), glutathione peroxidase (GSH-Px), glutathione reductase (GR) and glutathione S-transferase (GST) and the level of glutathione (GSH), and reduce the level of malondialdehyde (MDA) in rats induced by $\mathrm{CCl}_{4}$ intoxication. In addition, glycyrrhizic acid, 18ß-glycyrrhetinic acid, liquiritigenin, isoliquiritigenin, liquiritin and glabridin are the major bioactive components of licorice extract. Glycyrrhizic acid and 18 $\beta$-glycyrrhetinic acid (glycyrrhetinic acid) exhibit anti-inflammatory activities and neuroprotective effects; they also have significant effects on brain damage induced by ischemia and 6-hydroxydopamine. Recent studies have shown that glycyrrhizic acid and $18 \beta$ glycyrrhetinic acid can go through the blood-brain barrier (BBB). ${ }^{8}$ Glycyrrhizic acid can also suppress cell apoptosis, oxidative stress and inflammatory responses induced by lipopolysaccharides (LPS) in rat mesangial cells (HBZY-1) and septic rats. ${ }^{9}$ These results indicate that glycyrrhizic acid and 
$18 \beta$-glycyrrhetinic acid are potent agents for the treatment of neural diseases and Parkinson's disease. Liquiritigenin and isoliquiritigenin are chalconoids of licorice; liquiritin and isoliquiritin are the glycone forms of liquiritigenin and isoliquiritigenin, respectively. Liquiritin promotes neurite outgrowth in PC12 cells with nerve growth factor treatment, indicating its potential as a remedy for neurodegenerative diseases such as Alzheimer's disease or Parkinson's disease. ${ }^{\mathbf{1 0}}$ Liquiritin also exhibits an antidepressant-like effect in chronic variable stress-induced depression model rats by modulating oxidative stress. Liquiritigenin can promote the recovery of learning and memory deficits induced by amyloid beta $A \beta$ (25-35). ${ }^{11}$ Isoliquiritigenin promotes neuronal health by inhibiting monoamine oxidase $\mathrm{A}$ and B. Glabridin, an isoflavonoid found in licorice with positive antioxidant properties, has frequently been used in studies of oxidative stress. It can also significantly improve learning and memory impairment induced by diabetes in rats. ${ }^{12}$ In addition, glycyrrhizin can also ameliorate memory deficits, such as in Alzheimer's disease.

Almost all of these results provide evidence that licorice extract and its bioactive ingredients have the remarkable effects of antioxidation, anti-apoptosis and amelioration of learning and memory functions in rats. Additionally, now researchers believe that the antioxidant effect may be beneficial in delaying senescence, and many studies have demonstrated that antioxidant supplements may alleviate age-related cognition impairment and delay the process of aging. ${ }^{7-9}$ Although considerable evidence has shown that licorice has a remarkable antioxidative effect, little research has shown that licorice can have anti-aging effects in senescence-related models. The aim of the present study was to investigate whether licorice extract can improve oxidative damage and inhibit neuron apoptosis in D-gal induced aging models.

A large number of research studies have also indicated that D-gal induced aging rats have more similar natural aging characteristics, especially oxidative damage and a decline in cognitive ability. ${ }^{13-15}$ D-gal was administrated long-term to rats which led to massive free radical production and induced the occurrence of oxidative stress, which further resulted in neural dysfunction and the debasement of learning and memorizing ability. ${ }^{\mathbf{1 6}}$ Furthermore, excessive free radicals can modify the amino groups of proteins and peptides without enzymes to form complex advanced glycation end products (AGEs). AGEs can enhance oxidative stress damage, leading to changes in the physiological activity and physiological function. ${ }^{17-19}$ At the same time, studies have indicated that D-gal can also accelerate neuronal apoptosis and neurodegeneration, which are found in natural aging, and so it is widely used to establish the aging rat model. ${ }^{16,20-22}$

Therefore, in this study, we investigated the pharmacological effect and mechanism of licorice extract in improving learning and memory functions in D-gal induced aging rats. A considerable amount of data was provided to support the idea that licorice extract can ameliorate learning and memory ability and also to indicate the possible mechanisms in D-gal induced aging rats.

\section{Materials and methods}

\subsection{Reagents}

D-Gal was purchased from Sigma Chemical Co. (St. Louis, MO, USA) and dissolved in $0.9 \%$ saline at a concentration of $60 \mathrm{mg}$ $\mathrm{ml}^{-1}$. Licorice (glycyrrhiza uralensis Fisch) was produced in the Inner Mongolia Autonomous Region and purchased from the Changcheng drugstore in Taiyuan (Shanxi, China). The quality of the licorice accorded with the standard of the Chinese Pharmacopoeia (2015 edition). Commercial kits were used for determining the superoxide dismutase (SOD), acetyl cholinesterase (AChE), acetylcholine $(\mathrm{ACH})$, catalase (CAT), glutathione peroxidase (GSH-Px) and malondialdehyde (MDA) activities. They were purchased from Nanjing JianCheng Bioengineering Institute (Nanjing, China). Primary antibodies for $\beta$-actin, cytochrome C (Cyt-C), caspase-3, Bcl-2 and Bax were purchased from Cell Signaling (CST, USA).

\subsection{Extract preparation and LC-MS analysis}

Licorice was extracted twice, for $2 \mathrm{~h}$ each time, by refluxing in water. The combined solution was filtered and concentrated in a rotary evaporator and then freeze-dried to a powder under vacuum, and was stored at $4{ }^{\circ} \mathrm{C}$ until use. The yield of powder was $41.03 \%$ of the original dry materials. The constituents of the licorice extract were analyzed by ultra-performance liquid chromatography coupled with mass spectrometry (Thermo Scientific Exactive LC-MS, Thermo, USA) with an Acquity UPLC HSS T3 $(2.1 \mathrm{~mm} \times 100 \mathrm{~mm}, 1.8 \mu \mathrm{m})$. The composition of the licorice extract is shown in Table $\mathrm{S} 1 . \dagger$ The mass spectrometer was operated in both the positive ion detection mode (Fig. S1A $\dagger$ ) and the negative ion detection mode (Fig. S1B $\dagger$ ).

\subsection{Animals and drugs administration}

A total of 75 young adult male Sprague-Dawley rats (180 $\pm 20 \mathrm{~g}$ ) were purchased from Beijing Vital River Laboratory Animal Technology (Beijing, China) and employed in the study. The rats were housed in a well-ventilated animal experimental laboratory with a $12 \mathrm{~h}$ light/dark cycle, a constant temperature of $25 \pm$ $1{ }^{\circ} \mathrm{C}$, and a relative humidity of $50 \pm 10 \%$. The rats were randomly assigned to cages, 5 to each, with ad libitum access to a standard rodent diet and tap water. All of the animal experiments were carried out in accordance with the National Guidelines for Experimental Animal Welfare (MOST, PR China, 2006), which had full accreditation from the Association for Assessment and Accreditation of Laboratory Animal Care International (AAALAC Intl.). This study was approved by the Committee on the Ethics of Animal Experiments of Shanxi University. Maximal effort was made to minimize animal suffering and the number of animals necessary for the acquisition of reliable data.

After one week of acclimatization, the rats were randomly divided into five groups $(n=15)$ : a control and model group, and three licorice groups. The model group and the three licorice groups received D-gal at a dose of $300 \mathrm{mg} \mathrm{kg}^{-1}$ for 5 consecutive weeks while the control group received the same volume of physiological saline. At the same times, the three 
licorice groups were administered with licorice extract dissolved in water at doses of 1, 5 and $10 \mathrm{~g}$ of herb per $\mathrm{kg}$, respectively, by oral gavage after D-gal was injected intraperitoneally. The rats in the control group and the model group were administrated with the same volume of water. Body weight was examined weekly during the period of $\mathrm{D}$-gal administration to monitor the general health of all of the groups.

\subsection{Behavioral tests}

2.4.1 Morris water maze. The ability of rats to learn and remember the location of a hidden platform was determined by the Morris water maze (MWM) test, using our previously described protocol. In brief, the maze consisted of a large stainless steel circular pool $(100 \mathrm{~cm}$ in diameter and $60 \mathrm{~cm}$ in height), which was filled with water $\left(25^{\circ} \mathrm{C}\right)$ to a depth of $30 \mathrm{~cm}$. The pool was divided virtually into four equal quadrants, and a transparent platform (escape platform) was submerged $1.5 \mathrm{~cm}$ below the surface of the water in a fixed location. The spatial learning-memory test included a place navigation test and a probe test. In the place navigation test, each rat received 4 training trials with different starting positions each day for 5 consecutive days. After climbing onto the platform, the rat was permitted to remain there for $15 \mathrm{~s}$. If the rat failed to reach the platform within $60 \mathrm{~s}$, it was gently placed on the platform and allowed to remain there for $15 \mathrm{~s}$. The time taken to reach the platform (escape latency) was recorded. On day 6, the probe test without the platform was conducted with a cutoff time of $60 \mathrm{~s}$. The time spent in the target quadrant that previously contained the platform and the number of platform location crossings were recorded. Data acquisition was performed using a video camera (Viewer 2 Tracking Software, Ji Liang Instruments, China). ${ }^{23,24}$

2.4.2 Object-place recognition. Twenty-four hours after the Morris water maze test, each rat was trained and tested in the object-place recognition task. Before training, each rat was allowed to adapt for $10 \mathrm{~min}$ in an open field box $(70 \times 50 \times 40$ $\mathrm{cm}$ ), and then taken in intervals of $30 \mathrm{~min}$ to start training. The training and testing of the object recognition task took place in a box and the time given to each individual rat was 10 minutes. In the training, two identical plastic objects (objects A: $2.5 \times 2.5$ $\times 5 \mathrm{~cm}$ ) were placed in the box diagonally at a distance of about $10 \mathrm{~cm}$ from the side of the box. After the training session of each rat, the object was wiped with alcohol and dried. Twenty-four hours after training, the test was conducted by placing individual rats in the open field box for 10 minutes. Two different objects (objects A and B; cube B: $5 \times 5 \times 5 \mathrm{~cm}$ ) were positioned on the same side at about $10 \mathrm{~cm}$ from the walls. All of the objects presented similar textures, colors, and sizes, but distinctive shapes. In the trial and test, the time spent on each object by each animal was recorded as a measurable index. ${ }^{25,26}$

\subsection{Preparation of hippocampus homogenates}

After the behavioral tests, all of the rats were anesthetized and sacrificed. The hippocampi of 10 rats from each group were excised and immediately frozen individually in liquid nitrogen. Then the hippocampus of each rat was dissolved on ice and immersed in ice-cold physiological saline. A 10\% homogenate of the hippocampus was prepared. The supernatant was collected after centrifugation (3500 rpm for $10 \mathrm{~min}$ at $4{ }^{\circ} \mathrm{C}$ ) for various biochemical assays. All of the surgeries were performed under sodium pentobarbital anesthesia, and all efforts were made to minimize suffering.

\subsection{Biochemical assays}

The MDA and ACh content, as well as the SOD, GSH-Px, CAT and AChE activities, were determined using commercially available kits. Briefly, SOD was assayed based on its ability to decrease the oxidation of hydroxylamine to form a red product. The GSH-Px activity assay was performed by detecting the absorbance of a yellow product formed in the reaction between reduced glutathione and dithiobisnitrobenzoic acid. CAT was determined based on the fact that ammonium molybdate can rapidly terminate the degradation reaction of hydrogen peroxide catalyzed by CAT and react with the residual hydrogen peroxide to generate a yellow product. AChE was measured by detecting the absorbance of sym-trinitrobenzene formed in the color reactions between acetylcholine hydrolyzed choline and acetic acid; choline can react with hydrosulphonyl to generate sym-trinitrobenzene, which is a yellow product. ACh was assayed by detecting the absorbance of a brown product formed in the color reactions between ACh and the substrate of the test kit. All of the procedures completely complied with the manufacturer's instructions.

\subsection{Hematoxylin and eosin staining}

After the behavioral tests, all of the rats were anesthetized and sacrificed. The hippocampi of 5 rats from each group were fixed in $4 \%$ paraformaldehyde solution for $24 \mathrm{~h}$. After routine tissue processing, the tissues were embedded in paraffin. Then, $4 \mu \mathrm{m}$ thick sections obtained from each paraffin block were stained with hematoxylin and eosin (HE) stain for histopathological evaluation. Finally, histological features of apoptotic cells in the hippocampi revealed by the HE staining were observed under an optical microscope. ${ }^{27-29}$

\subsection{Western blot analysis}

The total proteins of the hippocampi were extracted, and the concentrations were measured by a BCA procedure. Samples containing $50 \mu \mathrm{g}$ proteins were separated by SDS-PAGE electrophoresis and transferred to PVDF membranes. The membranes were blocked with $5 \%$ bovine albumin (BSA) in Tris buffer saline-Tween 20 (TBST) for two hours at $37^{\circ} \mathrm{C}$ and then incubated overnight at $4{ }^{\circ} \mathrm{C}$ with the respective first antibodies diluted in $1.5: 1000$. After washing with TBST, the membranes were incubated with fluorescent secondary antibodies $(1: 15000)$ for $2 \mathrm{~h}$ at $37^{\circ} \mathrm{C}$. After rewashing with TBST, the membranes were scanned and visualized by a fluorescent scanner (Odyssey CLX, Gene company limited, USA). ${ }^{\mathbf{3 0}, 31}$ 


\subsection{Statistical analysis}

All of the results were normally distributed and are presented as mean \pm standard error of mean (SEM). In the case of multiple mean comparisons, the data were obtained using a one-way ANOVA, followed by a Tukey multiple comparison test. A $p$ value less than 0.05 was regarded as statistically significant. All of the analyses were carried out using a GraphPad Prism 5.0 vision. The results were considered statistically significant at $p<$ 0.05 .

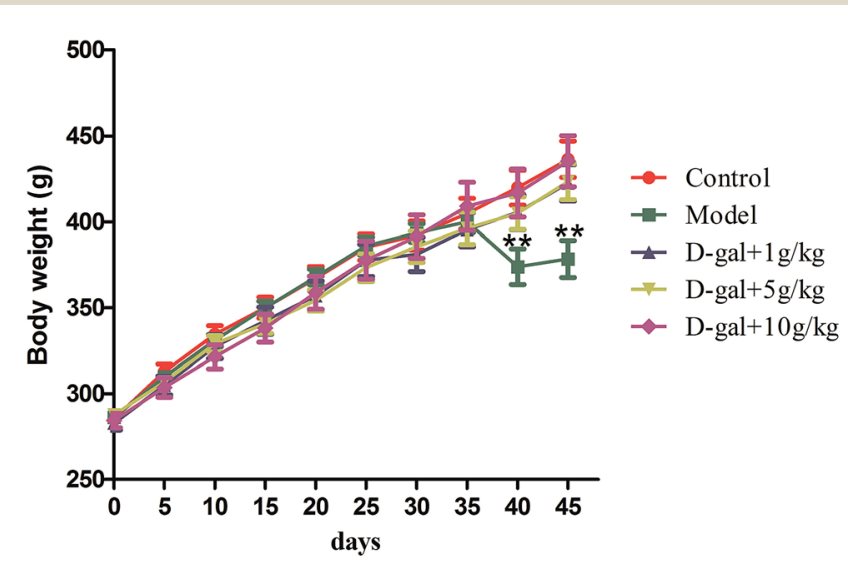

Fig. 1 Effect of the administration of licorice extract on the body weight of $D$-galactose treated rats. Values are expressed as mean \pm $\operatorname{SEM}(n=15)$.

\section{Results}

\subsection{Effect of licorice extract on body weight in D-gal treated} rats

In the present study, rats injected with D-gal (i.p., $300 \mathrm{mg} \mathrm{kg}^{-1}$ body weight) daily for five consecutive weeks experienced a statistically significant effect $(p<0.05)$ in bodyweight compared with those of the control group (Fig. 1), while consecutive licorice extract administration was able to reverse the animals' body weight loss. Licorice extract treated rats experienced a barely significant effect in body weight compared with the control rats. It was indicated that consecutive administration of licorice extract can improve the weight loss caused by the D-gal.

\subsection{Behavioral effects of the licorice extract}

3.2.1 Morris water maze. Cognitive behavioral deficit is one of the age-related markers of brain dysfunction. In the present study, the Morris water maze task was used to evaluate the spatial learning and memory ability of rats. The results showed that the D-gal treated rats had impaired cognition and memory as indicated by the extended escape latencies across the successive days $(p<0.001)$ (Fig. 2A), performed a reduced number of platform crossings $(p<0.05)$ (Fig. 2B) and spent a significantly decreased time in the goal area $(p<0.05)$ (Fig. 2C) compared with those of the control group during the test hidden sessions. However, rats in the D-gal treatment plus
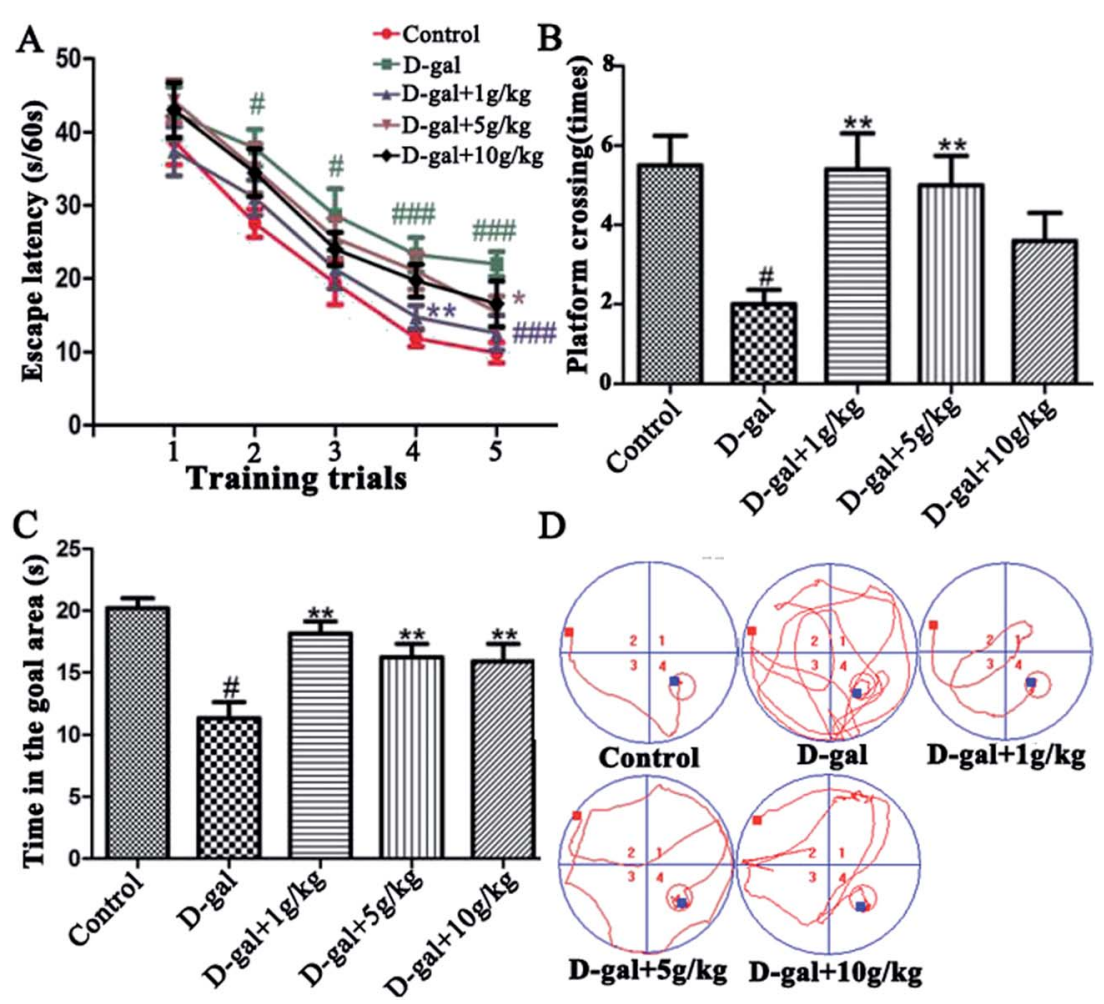

Fig. 2 Effects of licorice extract on D-gal induced cognitive impairment in the MWM test. (A) Escape latency within $60 \mathrm{~s}$ during the 5 day place navigation training, (B) the number of platform crossings in $60 \mathrm{~s}$ and $(\mathrm{C})$ the time spent in the goal area during the probe trial test were analyzed. (D) The representative traces on the last day of hidden platform training. All values given are the mean $\pm \operatorname{SEM}(n=15) .{ }^{\#} p<0.05,{ }^{\# \#} p<0.01$ and $\# \#$ \#\# 0.001 vs. control group. ${ }^{*} p<0.05,{ }^{* *} p<0.01$ and $* * * p<0.001$ vs. the model group. 
A

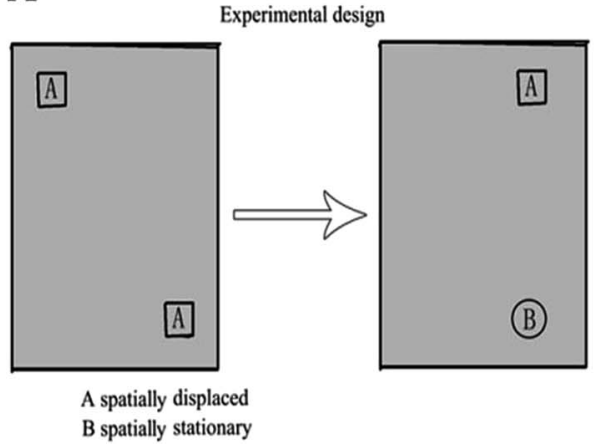

B

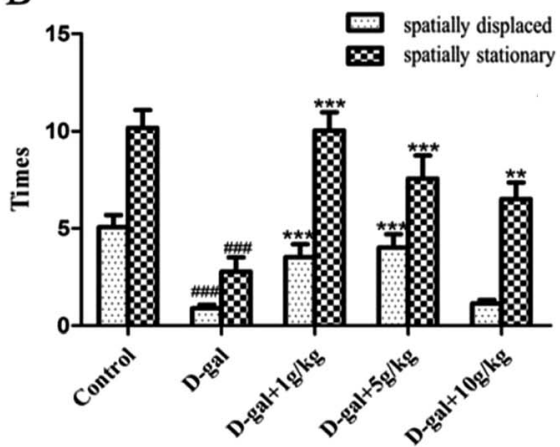

Fig. 3 Effects of licorice extract on D-gal induced cognitive impairment in object-place recognition. The data are represented as the mean plus the SEM of the object exploration time (seconds) of spatially stationary and spatially displaced objects in the test trial.All values given are the mean \pm SEM $(n=15) .{ }^{\# \# \#} p<0.001$ vs. control group. ${ }^{* *} p<0.01$ and $* * * p<0.001$ vs. the model group.

licorice extract administration group displayed remarkable increases in the number of platform crossings $(p<0.01)$ (Fig. 2B) and the time spent in the goal area $(p<0.01)$ (Fig. 2C), and reduced escape latencies in the probe test $(p<0.05)$ (Fig. 2A) compared to those of the model group. The representative traces on the last day of hidden platform training are shown in Fig. 2D. These results indicate that the D-gal treated rats had impaired spatial learning and memory, while the administration of licorice extract could recover the age-related cognitive impairment caused by D-gal.

3.2.2 Object-place recognition test. Results of the objectplace recognition test for all of the groups are presented in Fig. 3. The model group showed a significant deficit in object recognition learning compared with the control group. In contrast, the rats with licorice extract showed a significant improvement in learning and memory in the object-place recognition task (Fig. 3A) compared with those of the model group (Fig. 3B). These results suggest that licorice extract administration can significantly ameliorate the memory ability of D-gal induced aging rats for spatially displaced objects and stationary objects.

\subsection{Effects of licorice extract on anti-oxidation activities}

The SOD, CAT, and GSH-Px activities and the MDA content in the hippocampal tissue were determined as the biomarkers of oxidative stress in D-gal induced aging rats. These results (Fig. 4) indicate that D-gal was consecutively received and induced a significant decrease in the SOD, CAT and GSH-Px activities (Fig. 4A-C) and increase in the MDA level (Fig. 4D) compared those of the control group. All of the licorice groups had a significant decrease in MDA level compared with that of the model group. A significant increase in SOD, CAT and GSH-Px activity was observed in the licorice extract administered $(1 \mathrm{~g}$ herb per kg) group compared with those of the model group $\left({ }^{*} p\right.$ $<0.05,{ }^{*} p<0.05$, and ${ }^{* * *} p<0.05$, respectively).

\subsection{Effects of licorice extract on AChE activities and ACh content}

The AChE activity in the hippocampal tissue was increased in the D-gal treated rats compared with that of the control group $\left({ }^{\# \#} p<0.001\right)$ and this is shown in Fig. 5A, while the level of ACh in the hippocampal tissue was decreased in the D-gal treated rats compared with that of the control group $\left({ }^{\#} p<0.05\right)$ and this is shown in Fig. 5B. D-Gal treatment may induce a disorder in the cholinergic system. Licorice extract administration at a dose of $1 \mathrm{~g}$ of herb per $\mathrm{kg}$ significantly reversed the abnormalities in the AChE and ACh activities compared with those of the control group (Fig. 5). This result indicated that licorice extract might ameliorate dysfunction of the cholinergic system.
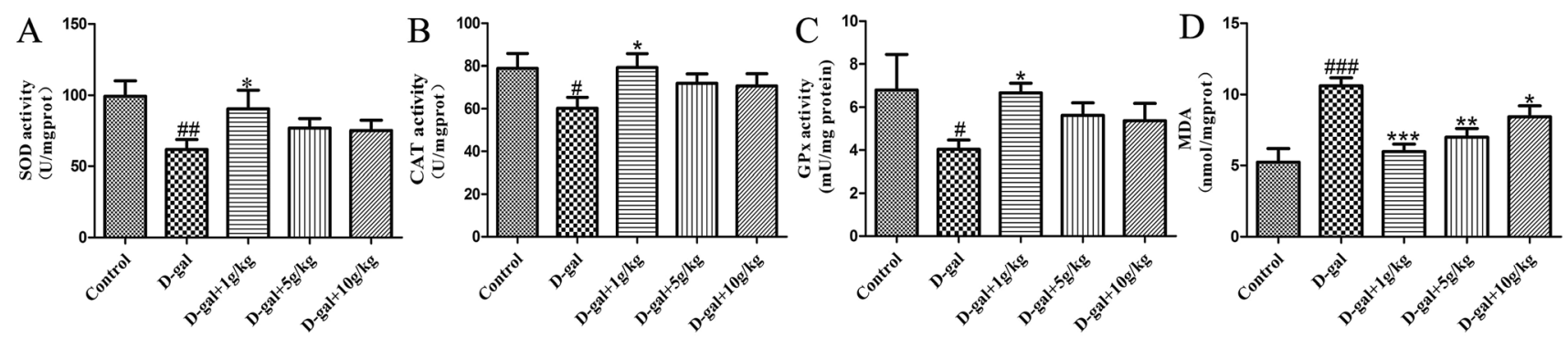

Fig. 4 Effect of licorice extract on SOD (A), CAT (B) and GPx (C) activity and MDA (D) level in the hippocampi of D-gal-treated rats. Values are

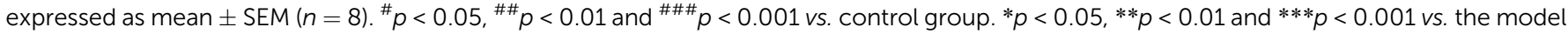
group. 

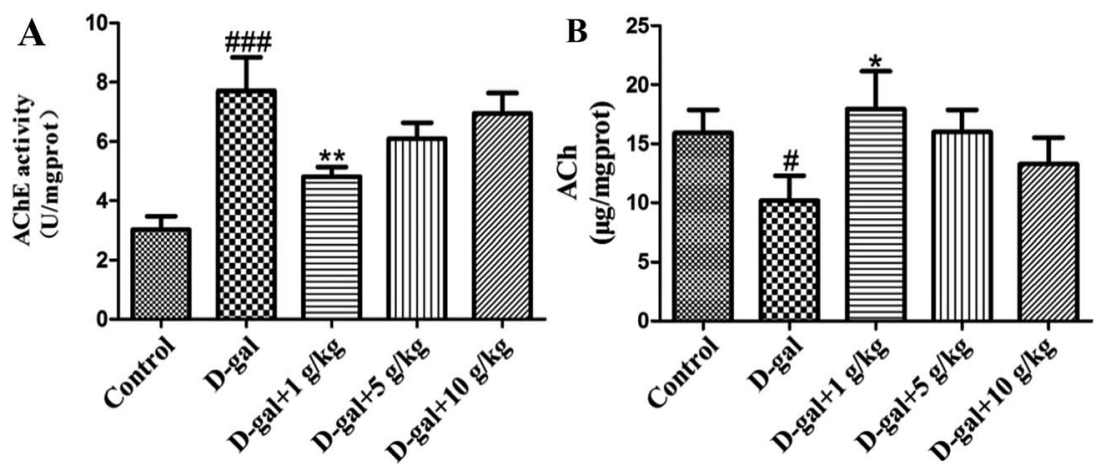

Fig. 5 Effect of licorice extract on AChE activity (A) and ACh content (B) in the hippocampi of D-gal-treated rats. Values are expressed as mean \pm SEM $(n=8) .{ }^{\#} p<0.05$ and ${ }^{\# \# \#} p<0.001$ vs. the control group. ${ }^{*} p<0.05$ and $* * p<0.01$ vs. the model group.

\subsection{Histopathological observation of the neurons in the hippocampus}

HE staining of features of cell loss in the hippocampus is shown in Fig. 6. Rats in the control group had full hippocampal neurons, which were arranged tightly and were morphologically intact. The pyramidal neurons presented round and large nuclei and clear nucleoli (Fig. 6A). Widespread damage was visible in the hippocampi of the model group treated with D-gal (Fig. 6B).
The intercellular space increased in size, and the cells were loosely arranged. The pyramidal neurons either presented a densely stained shrunken appearance with minimal cytoplasm or disappeared. However, when licorice extract was administered after the D-gal was injected, the neuronal cells in the hippocampus were rescued (Fig. 6C-E) compared with those of the model group. The neurons in the hippocampi of the rats treated with licorice extract at a dose of $1 \mathrm{~g}$ of herb per $\mathrm{kg}$ appeared almost normal (Fig. 6C).
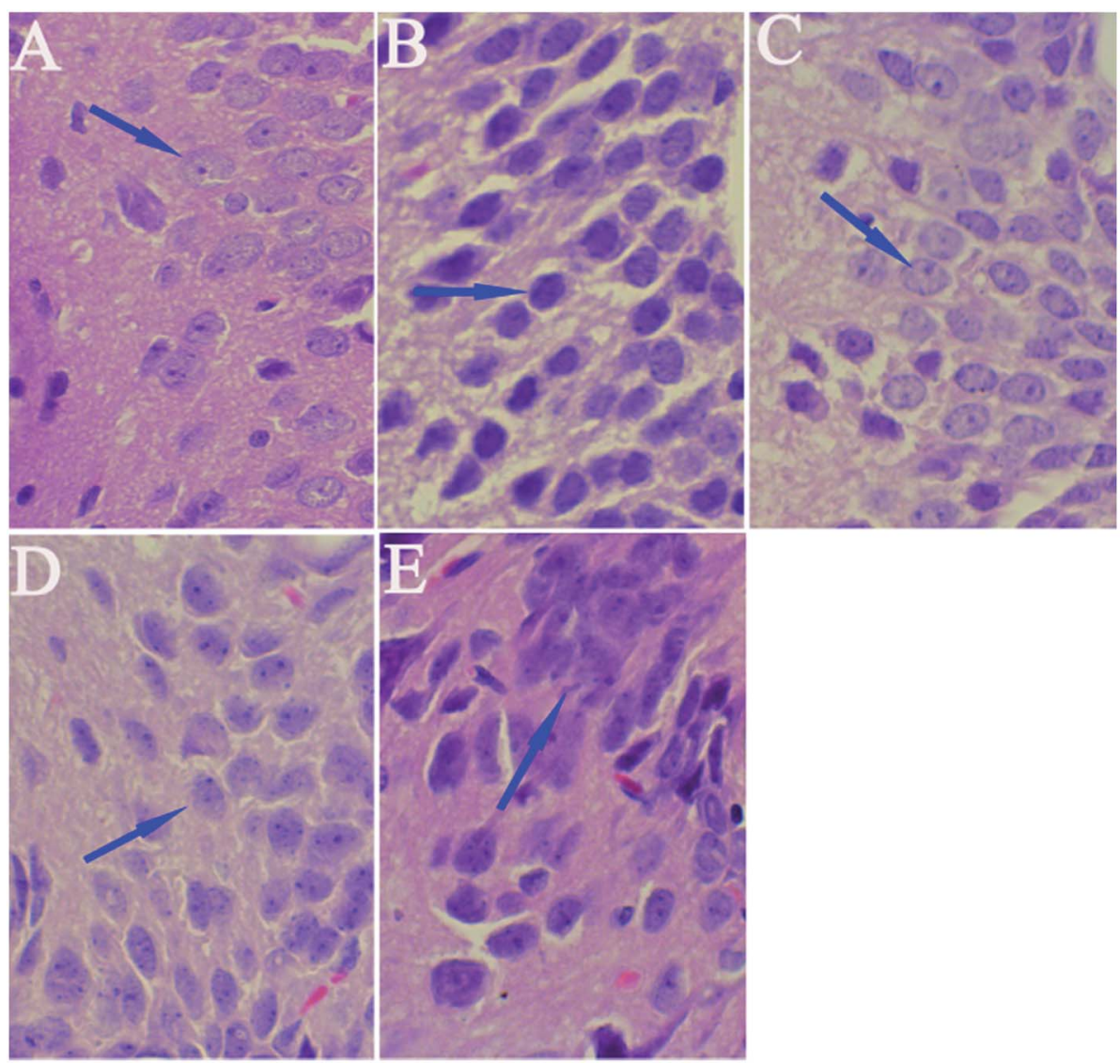

Fig. 6 Protective effect of licorice extract on cell loss in the hippocampi of $D$-gal-treated rats revealed by hematoxylin and eosin (HE) staining (×200). Note: (A) control group; (B) model group; (C) D-gal $+1 \mathrm{~g} \mathrm{~kg}^{-1}$ licorice extract group; (D) D-gal $+5 \mathrm{~g} \mathrm{~kg}{ }^{-1}$ licorice extract group; (E) D-gal + $10 \mathrm{~g} \mathrm{~kg}^{-1}$ licorice extract group. 

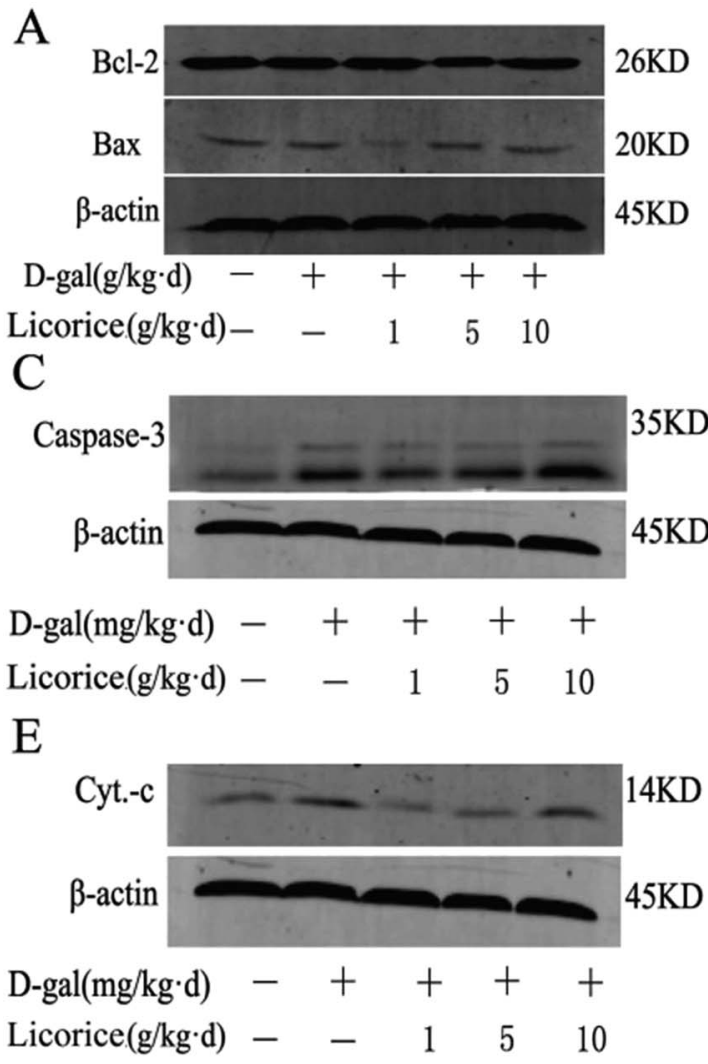

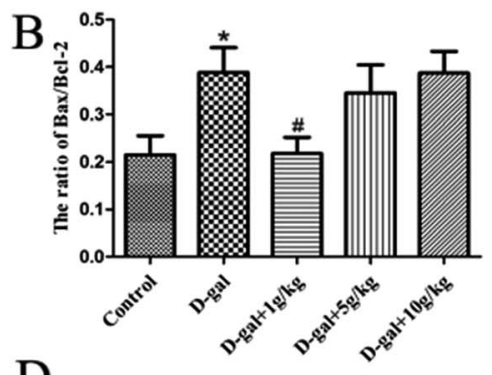

$\mathrm{D}$
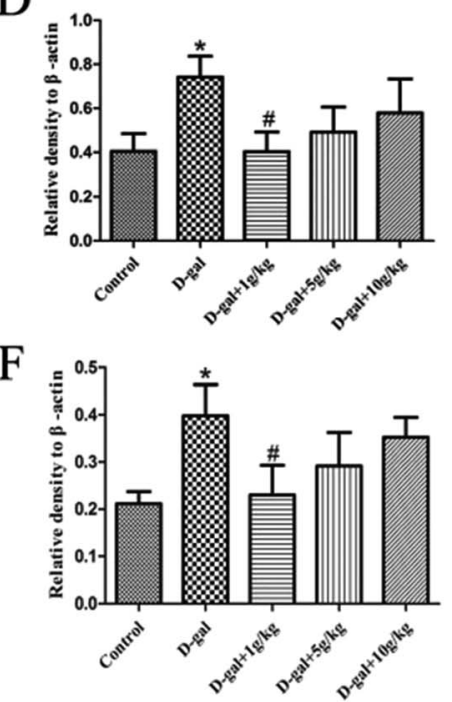

Fig. 7 Effect of licorice extract on the expression levels of apoptosis-related proteins. Bax/Bcl-2 ratio (A/B), caspase-3 (C/D) and Cyt-C (E/F) induced by $D$-gal are expressed as a ratio of $\beta$-actin. All values given are the mean $\pm \operatorname{SEM}(n=4) .{ }^{*} p<0.05 \mathrm{vs}$. the control group and ${ }^{*} p<0.05 \mathrm{vs}$. the model group.

\subsection{Effects of licorice extract on expression levels of} apoptosis-related proteins including Bax, Bcl-2, caspase-3 and Cyt-C

Bcl-2 is an anti-apoptotic protein, and Bax, Cyt-c and caspase-3 are pro-apoptotic proteins. It is documented that the $\mathrm{Bax} / \mathrm{Bcl}-2$ ratio, and the caspase- 3 and Cyt-C levels are crucial mediators for aging progression. To further explore the neuroprotective mechanism of licorice extract in D-gal treated rats, a western blot was used to detect the protein levels of Bax, Bcl-2, caspase-3 and Cyt-c in the hippocampus. The western blot analysis revealed that D-gal led to a significant increase in the Bax/Bcl-2 ratio (Fig. 7A and B), and the caspase-3 (Fig. 7C and D) and Cyt-C (Fig. 7E and F) levels in the model group compared with those of the control group. Licorice extract administration remarkably reduced the Bax/Bcl-2 ratio, and the caspase- 3 and Cyt-C levels compared with those of the D-gal treated rats. These results indicate that licorice extract has the effects of anti-apoptosis and neuroprotection through regulating the expression of apoptosis-related proteins.

\section{Discussion}

There has been a lot of research indicating that senescence is associated with oxidative stress, neurodegeneration and cell apoptosis and is accompanied by memory and cognitive decline. ${ }^{32-34}$ The model group rats were continuously treated with D-gal, which could produce a process similar to natural aging. ${ }^{35}$ In the present study, brain aging characteristics induced by D-gal were assessed by the Morris water maze and object-place recognition. When the rats in the administration group were treated with licorice extract long-term, memory impairment, oxidative stress, neuronal apoptosis and cholinergic dysfunction were all improved. According to the oxidative stress theory of aging, oxidative stress is the disturbance of the balance of oxidation and anti-oxidation in vivo. Reactive Oxygen Species (ROS) are produced as a normal product of aerobic metabolism. While ROS are excessively generated and exceed the ability of organism anti-oxidation the body will be in oxidative stress, which leads to a sharp deterioration of many bodily functions such as a gradual deterioration in learning and memory capacity and acceleration of apoptosis levels in the brain. ${ }^{\mathbf{3 6}}$ There are numerous endogenous antioxidants (SOD, GSH-Px, and CAT) that constitute the body's own natural defense systems and keep a certain balance with ROS to limit free radical damage in neuronal tissues. ${ }^{37}$ The oxidative stress damage increases in aging, mainly because of anti-oxidation deficiency. ${ }^{38}$ We found that licorice extract could reduce MDA levels and enhance antioxidant activities, such as the activities of SOD, CAT and GSH-Px. 
The cholinergic system plays a significant role in learning and memory. Increased activity of the cholinergic system of the basal forebrain of the hippocampus is thought to contribute to an improvement in cognitive function impairment. Acetylcholine (ACh) is an important neurotransmitter involved in learning and memory. Acetylcholinesterase (AChE) is responsible for the degradation of ACh into acetate and choline in the synaptic cleft (Qu et al. 2016.) Numerous studies have shown that rats treated with D-gal exhibit a significant increase in AChE activity and a decrease in ACh activity. ${ }^{39}$ When licorice extract was administered at a dose of $1 \mathrm{~g}$ of herb per $\mathrm{kg}$, the AChE activities and ACh content were significantly recovered.

Several studies have also shown that neurodegenerative dysfunction and cell apoptosis are also involved in age-related processes. $^{40}$ Memory and learning loss and neuronal apoptosis are the major neuropathological hallmarks of aging. Neurodegenerative dysfunction and cell apoptosis play a crucial role in senescence. Without obvious cause, a slow progression of symptoms, deterioration of the memory step-by-step, cognitive dysfunction, personality change and progressive irreversibility are the characteristics of neurodegenerative dysfunction. $^{41}$ The pathological fundament is nerve cell degeneration or death. D-Gal can accelerate the apoptosis of neurons and inhibit the formation of neurons through caspase. So HE staining of the hippocampal tissue was performed. The results showed that licorice extract has a significant neuroprotective effect. In order to further support this result, some apoptosis-related protein expression in the hippocampus was detected by western blot. Bcl-2 family members have an affect on cell apoptosis by changing the permeability of the mitochondrial membrane. The main characteristic of the mitochondria-mediated apoptosis pathway is mitochondrial dysfunction with the release of Cyt-C, followed by formation of apoptotic bodies, which in turn causes activation of caspase-3. Caspase-3 is one of the key proteases responsible for the initiation of the caspase cascade which leads to apoptosis. ${ }^{42,43}$ The results of the western blot analysis also showed that licorice extract ( $1 \mathrm{~g}$ of herb per $\mathrm{kg}$ ) has a neuroprotective effect on the rat aging model induced by D-gal.

\section{Conclusions}

In summary, the results of this study demonstrate that chronic administration of a high dose of D-gal can induce significant neurobehavioral deficits and consequent damage of hippocampal neurons by triggering apoptosis cascades, oxidative stress and cholinergic system disorder. Licorice extract $(1 \mathrm{~g}$ of herb per $\mathrm{kg}$ ) could prevent these effects by enhancing cognitive performance, regulating oxidative stress and the cholinergic system, and displaying anti-apoptotic effects by decreasing the Bax/Bcl-2 ratio, and the levels of caspase- 3 and Cyt-C. Taken together, these data suggest that licorice extract may be a beneficial candidate for the treatment of aging-related conditions.

\section{Conflicts of interest}

There are no conflicts to declare.

\section{Acknowledgements}

We acknowledge financial support from the Applied Basic Research Project of Shanxi Province (No. 201601D021164), the Innovation Project of Higher Education Institutions in Shanxi Province (No. 2016120), the Construction of the Science and Technology Basic Condition Platform of Shanxi Province (No. 2014091022), and the Program of Science and Technology of Shanxi Province (No. 20140313008-14).

\section{References}

1 T. C. Kao, C. H. Wu and G. C. Yen, J. Agric. Food Chem., 2014, 62, 542-553.

2 S. Fujii, O. Morinaga, T. Uto, S. Nomura and Y. Shoyama, J. Agric. Food Chem., 2016, 64, 1087-1093.

3 H. J. Chen, S. P. Kang, I. J. Lee and Y. L. Lin, J. Agric. Food Chem., 2014, 62, 618-625.

4 S. Ji, Z. Li, W. Song, Y. Wang, W. Liang, K. Li, S. Tang, Q. Wang, X. Qiao, D. Zhou, S. Yu and M. Ye, J. Nat. Prod., 2016, 79, 281-292.

5 Y. M. Kim, T. H. Kim, Y. W. Kim, Y. M. Yang, D. H. Ryu, S. J. Hwang, J. R. Lee, S. C. Kim and S. G. Kim, Free Radical Biol. Med., 2010, 49, 1722-1734.

6 S. M. Park, J. R. Lee, S. K. Ku, I. J. Cho, S. H. Byun, S. C. Kim, S. J. Park and Y. W. Kim, Eur. J. Nutr., 2016, 55, 2431-2444.

7 H. Z. Huo, B. Wang, Y. K. Liang, Y. Y. Bao and Y. Gu, Int. J. Mol. Sci., 2011, 12, 6529-6543.

8 M. Tabuchi, S. Imamura, Z. Kawakami, Y. Ikarashi and Y. Kase, Cell. Mol. Neurobiol., 2012, 32, 1139-1146.

9 H. Zhao, Z. Liu, H. Shen, S. Jin and S. Zhang, Eur. J. Pharmacol., 2016, 781, 92-99.

10 R. T. Liu, L. B. Zou, J. Y. Fu and Q. J. Lu, Behav. Brain Res., 2010, 210, 24-31.

11 Z. A. Chen, J. L. Wang, R. T. Liu, J. P. Ren, L. Q. Wen, X. J. Chen and G. X. Bian, Cytotechnology, 2009, 60, 125-132.

12 P. Hasanein, Acta Physiol. Hung., 2011, 98, 221-230.

13 J. Budni, R. Pacheco, S. da Silva, M. L. Garcez, F. Mina, T. Bellettini-Santos, J. de Medeiros, B. C. Voss, A. V. Steckert, S. Valvassori Sda and J. Quevedo, Behav. Brain Res., 2016, 302, 35-43.

14 J. Gao, H. He, W. Jiang, X. Chang, L. Zhu, F. Luo, R. Zhou, C. Ma and T. Yan, Behav. Brain Res., 2015, 293, 27-33.

15 T. Ali, H. Badshah, T. H. Kim and M. O. Kim, J. Pineal Res., 2015, 58, 71-85.

16 W. J. Li, S. P. Nie, X. P. Peng, X. Z. Liu, C. Li, Y. Chen, J. E. Li, W. R. Song and M. Y. Xie, J. Agric. Food Chem., 2012, 60, 1413-1418.

17 S. Haider, L. Liaquat, S. Shahzad, S. Sadir, S. Madiha, Z. Batool, S. Tabassum, S. Saleem, F. Naqvi and T. Perveen, Life Sci., 2015, 124, 110-119.

18 F. Ullah, T. Ali, N. Ullah and M. O. Kim, Neurochem. Int., 2015, 90, 114-124.

19 X. Song, M. D. Bao and Y. Li, Mech. Ageing Dev., 1999, 108, 239-251. 
20 A. F. Aydin, J. Coban, I. Dogan-Ekici, E. Betul-Kalaz, S. Dogru-Abbasoglu and M. Uysal, Metab. Brain Dis., 2016, 31, 337-345.

21 X. Li, Y. Chen, S. Shao, Q. Tang, W. Chen, Y. Chen and X. Xu, Exp. Gerontol., 2016, 83, 89-93.

22 Z. F. Zhang, S. H. Fan, Y. L. Zheng, J. Lu, D. M. Wu, Q. Shan and B. Hu, J. Agric. Food Chem., 2009, 57, 7731-7736.

23 J. Gao, R. Zhou, X. You, F. Luo, H. He, X. Chang, L. Zhu, X. Ding and T. Yan, Metab. Brain Dis., 2016, 31, 771-778.

24 H. Fukami, H. Tachimoto, M. Kishi, T. Kaga and Y. Tanaka, J. Agric. Food Chem., 2010, 58, 4084-4089.

25 M. A. de Souza Silva, B. Lenz, A. Rotter, T. Biermann, O. Peters, A. Ramirez, F. Jessen, W. Maier, M. Hull, J. Schroder, L. Frolich, S. Teipel, O. Gruber, J. Kornhuber, J. P. Huston, C. P. Muller and S. Schable, Proc. Natl. Acad. Sci. U. S. A., 2013, 110, 15097-15102.

26 M. Leger, A. Quiedeville, V. Bouet, B. Haelewyn, M. Boulouard, P. Schumann-Bard and T. Freret, Nat. Protoc., 2013, 8, 2531-2537.

27 M. S. Rofiee, M. I. Yusof, E. E. Abdul Hisam, Z. Bannur, Z. A. Zakaria, M. N. Somchit, L. K. Teh and M. Z. Salleh, J. Ethnopharmacol., 2015, 166, 109-118.

28 P. P. Wang, H. X. Sun, C. J. Liu, M. H. Hu, X. Q. He, S. Yue, Z. Z. Jiao and L. Xiang, Phytomedicine, 2016, 23, 460-467.

29 Z. Xiang, H. Sun, X. Cai, D. Chen and X. Zheng, Mol. BioSyst., 2015, 11, 1067-1078.

30 T. Wang, G. Di, L. Yang, Y. Dun, Z. Sun, J. Wan, B. Peng, C. Liu, G. Xiong, C. Zhang and D. Yuan, J. Pharm. Pharmacol., 2015, 67, 1284-1296.
31 Y. Wang, F. Huang, L. Zhao, D. Zhang, O. Wang, X. Guo, F. Lu, X. Yang, B. Ji and Q. Deng, J. Agric. Food Chem., 2016, 64, 161-170.

32 Q. Ruan, F. Liu, Z. Gao, D. Kong, X. Hu, D. Shi, Z. Bao and Z. Yu, Mech. Ageing Dev., 2013, 134, 89-97.

33 M. Kim, K. H. Cho, M. S. Shin, J. M. Lee, H. S. Cho, C. J. Kim, D. H. Shin and H. J. Yang, Int. J. Mol. Med., 2014, 33, 870-878.

34 Y. Garcia-Mesa, S. Colie, R. Corpas, R. Cristofol, F. Comellas, A. R. Nebreda, L. Gimenez-Llort and C. Sanfeliu, J. Gerontol., Ser. A, 2016, 71, 40-49.

35 Z. Du, S. Li, L. Liu, Q. Yang, H. Zhang and C. Gao, Mol. Med. Rep., 2015, 12, 7883-7890.

36 A. Iriondo-DeHond, P. Martorell, S. Genoves, D. Ramon, K. Stamatakis, M. Fresno, A. Molina and M. D. Del Castillo, Molecules, 2016, 21, E721.

37 Y. Lei, J. Chen, W. Zhang, W. Fu, G. Wu, H. Wei, Q. Wang and J. Ruan, Food Chem., 2012, 135, 2702-2707.

38 G. M. Cunningham, M. G. Roman, L. C. Flores, G. B. Hubbard, A. B. Salmon, Y. Zhang, J. Gelfond and Y. Ikeno, Arch. Biochem. Biophys., 2015, 576, 32-38.

39 H. Wei, Z. Gao, L. Zheng, C. Zhang, Z. Liu, Y. Yang, H. Teng, L. Hou, Y. Yin and X. Zou, Mar. Drugs, 2017, 15, 77.

40 C. Ntsapi and B. Loos, Exp. Gerontol., 2016, 83, 97-111.

41 S. Jamwal and P. Kumar, Physiol. Behav., 2016, 155, 180-187. 42 S. L. Sun, L. Guo, Y. C. Ren, B. Wang, R. H. Li, Y. S. Qi, H. Yu, N. D. Chang, M. H. Li and H. S. Peng, Mol. Biol. Rep., 2014, 41, 6117-6124.

43 S. Liang, K. Sun, Y. Wang, S. Dong, C. Wang, L. Liu and Y. Wu, Chem.-Biol. Interact., 2016, 258, 40-51. 